\title{
On the Conduction Mechanism of Silicate Glass Doped by Oxide Compounds of Ruthenium (Thick Film Resistors)
}

\section{-Diffusion and Percolation Levels}

\section{Gulmurza Abdurakhmanov}

The Institute of Power Engineering and Automation of the Uzbek Academy of Sciences, Tashkent, Uzbekistan.

Email: gulmirzo@mail.ru

Received March 15 ${ }^{\text {th }}$, 2011; revised March 23 ${ }^{\text {rd }}$, 2011; accepted March 25 ${ }^{\text {th }}, 2011$.

\begin{abstract}
The results of the investigation of conduction mechanism of silicate glass doped by oxide compounds of ruthenium (thick film resistor) are reported. The formation of diffusion zones in the softened glass during firing process of the mixture of the glass and the dopant powders is considered. As the result the doping glass becomes conductive. These diffusion zones have higher conductivity and act as percolation levels for the free charge carriers. The effect of temperature and duration of firing process on the conductivity of doped glass is considered. Experimental results are in a good agreement with the model.
\end{abstract}

Keywords: Lead-Silicate Glass, Thick Film Resistors, Percolation Levels, Doping, Conductivity, Firing Conditions

\section{Introduction}

The silicate glass doped by oxide compounds of ruthenium (DSG) and as a result becoming an electronic conductor is a functional material in thick film resistors [1], sensors [2] and electric heaters [3]. DSG is widely known as thick film resistors (TFR) but this name does not characterize DSG as a material. Despite the wide use of DSG, the mechanism of electrical conduction is not well understood yet.

For example variable range hopping, tunneling through potential barrier, thermal activation, effective medium approach and combinations of them have been exploited [4-8] to explain the temperature dependence of the DSG resistivity $\rho(T)$, schematically shown in Figure 1. Unfortunately these models can describe $\rho(T)$ in the narrow range of low temperatures (region I in Figure 1) only.

The quadratic temperature dependence

$$
\rho(T)=A\left(T-T_{\min }\right)^{2}+\rho_{m}
$$

is observed often in DSG in the region II, where $\rho_{\mathrm{m}}$ and $T_{\min }$ are the resistivity and the temperature at the minimum.
The quadratic dependence (1) and region III we have investigated here [9] do not correspond to any known models of the electrical conductivity of DSG.

There are quite a few questions which can not be understood in the framework of the above-mentioned models: how size and conductivity of the dopant particles affect the conductivity of DSG, the mechanism by which it is affected by temperature $T_{\mathrm{f}}$ and the duration $\tau$ of the firing on the room temperature value $\rho_{0}$ and slope of the $\rho(T)$, why the glass composition affects the conduction of the DSG, why the percolation threshold on the $\rho(C)$ shift or disappearance for glass of various content and etc. The $C=V_{\mathrm{d}} /\left(V_{g}+V_{\mathrm{d}}\right)$ here is the volume fraction of the dopant in DSG or the doping level, $V_{\mathrm{d}}$ and $V_{g}$ - volumes of the dopant and the glass accordingly, $V_{0}=V_{\mathrm{d}}+V_{g}$ is the total volume of the specimen.

We examine these questions while ignoring the role of the glass in conductance of the DSG. In fact at standard firing conditions (firing temperature $T_{\mathrm{f}} \approx 1123 \mathrm{~K}$ ) the lead-silicate glass softens and becomes very aggressive substance, enable to solve appreciable amounts of oxides of many metals (units or tens of \%) [10]. So long as fir- 


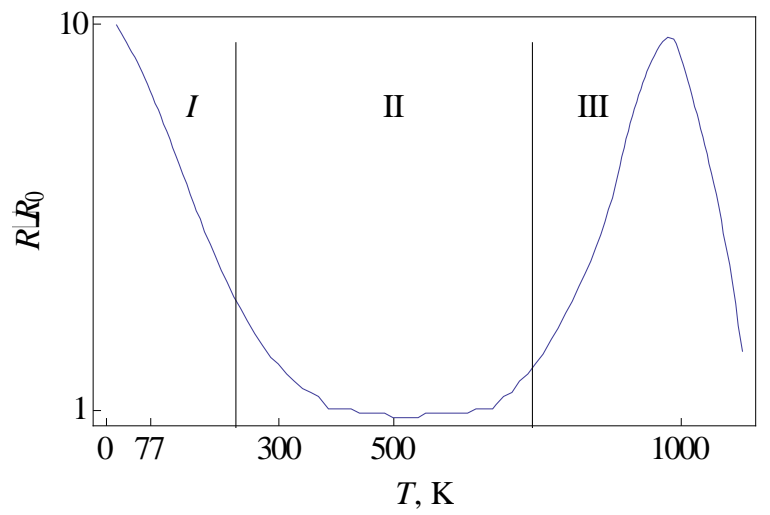

Figure 1. Resistivity vs temperature for DSG (schematically). Regions I, II and III correspond to low, middle and high temperature.

ing duration of DSG is limited (usually $\tau \approx 10 \mathrm{~min}$ ) and mechanical mixing is not affecting the distribution of solved atoms of the dopant in the glass, it should be diffusive.

In this paper we will consider a possible effect of diffusion of dopant atoms at $T_{\mathrm{f}}$ in the glass on the electrical conduction of the DSG.

The production of specimens is a standard procedure for thick film resistors and is described in many papers and books (see, for example, [2]). The glass compositions we used are as follows (weight \%):

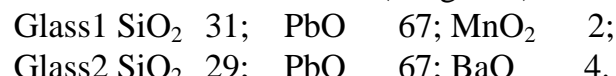

The DSG specimens compositions are as follows (weight \%):
1) Glass 1
80; $\quad \mathrm{Pb}_{2} \mathrm{Ru}_{2} \mathrm{O}_{6}$
20;
2) Glass 1
90; $\mathrm{RuO}_{2}$
10
3) Glass2 90; $\mathrm{RuO}_{2}$
10
4) Glass2 80; $\mathrm{RuO}_{2}$
20.

Volume fraction of dopant $C$ is less than theoretical percolation threshold (critical value $C_{\mathrm{c}}$ ) in all cases.

\section{Diffusion of Dopant Atoms into the Glass and Formation of Percolation Levels}

It should be noted that the percolation theory is considered as most common model of conduction mechanism of the DSG [11-13]. In this theory transport of charge takes place along the contiguous chains of intimately contacted dopant particles (infinite cluster) and the resistivity of DSG depends on the dopant volume fraction $C$ as

$$
\rho(C)=\rho_{0}\left(C-C_{\mathrm{C}}\right)^{-t}
$$

where $\rho_{0}$ is material-dependent prefactor, $C_{\mathrm{C}}$ is the percolation critical volume fraction of the dopant below which $\rho$ goes to infinity, $t$ is the dc transport critical exponent. According to the standard theory of transport percolation [12], $\quad C_{\mathrm{C}} \approx 0.12-0.39$ and $t \simeq t_{0} \simeq 1.6 \div 2$ is the universal value for three-dimensional disordered composites.

The expression (2) predicts percolation threshold of $\rho(C)$ at $C \approx C_{\mathrm{C}}$, while in reality the value of $t$ might be as high as 7 for various compositions of glass and doping conditions [14]. Additionally, the value of $C_{\mathrm{C}}$ is essentially lower than the theoretical estimation in many cases or percolation threshold disappears.

The reason for this discrepancy between the experimental results and the theory of percolation is the assumption that the volume fraction $C$ of the dopant in the DSG (after firing) is known a priori (often it equals to the initial volume fraction of them). In fact the interaction of dopant and the glass is possible during the firing process as well as the mutual diffusion of the glass and dopant atoms. As the result one does not have information about $C$ in the final stage of technological process and about the value of the resistivity of doped regions of the glass. So we should consider that the glass resistivity (in the diffusion zone) is reduced by many orders of magnitude due to diffusion (as in crystalline semiconductors) and infinite cluster consists of closed or overlapping diffusion zones which have been formed around the each dopant particle (Figure 2).

Let us to consider [15] that the diffusion zone is spherical because the glass is isotropic. This diffusion zone has the volume

$$
V_{\mathrm{d}}=\frac{4 \pi}{3} R_{\mathrm{d}}^{3}
$$

There $R_{\mathrm{d}}=r+l_{\mathrm{d}}$ is the radius of the diffusion zone, $r$ is the main radius of the dopant particles, and the diffusion length is

$$
l_{\mathrm{d}}=\sqrt{D \tau}=\sqrt{D_{0} \tau} \exp \left(-\frac{E_{\mathrm{d}}}{2 k T_{\mathrm{f}}}\right) .
$$

There $D$ is the diffusion coefficient, $D_{0}=D\left(T_{\mathrm{f}} \rightarrow \infty\right)$, $E_{\mathrm{d}}$ is the activation energy of diffusion, and $k$ is the Boltzmann constant. Inserting

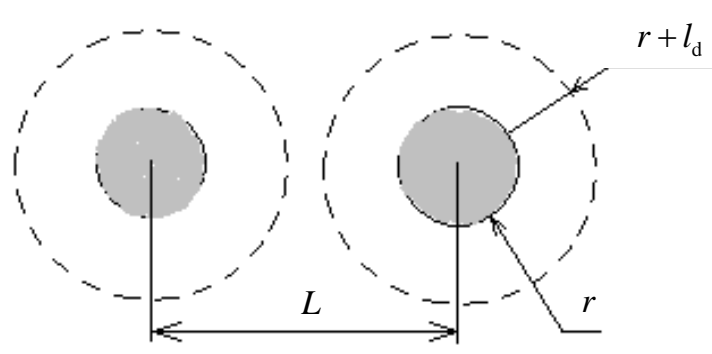

Figure 2. Formation of diffusion zone around the dopant particle of the radius $r$. There $r+l_{d}$ is the radius of diffusion zone, $l_{\mathrm{d}}$ is the diffusion length. $L$ is the main distance between the neighbor dopant particles in the DSG. 
$C=V_{\mathrm{d}} /\left(V_{\mathrm{d}}+V_{g}\right)$ and $C_{c}=V_{\mathrm{c}} /\left(V_{\mathrm{d}}+V_{g}\right)$ into (2) and taking into account (3) and (4) gives

$$
\begin{aligned}
& \ln \left\{\left[\frac{3 V_{0}}{4 \pi}\left(\left(\rho / \rho_{0}\right)^{-1 / t}+V_{c} / V_{0}\right)\right]-r\right\}= \\
& -\frac{E_{\mathrm{d}}}{2 k T_{\mathrm{f}}}+\frac{1}{2} \ln \left(D_{0} \tau\right) .
\end{aligned}
$$

Expression (5) for $\rho\left(T_{\mathrm{f}}\right)$ of DSG is in a good agreement with experiment (Figure 3) for various glasses and dopants and makes it possible to evaluate $D_{0}$, $E_{\mathrm{d}}$ and $l_{\mathrm{d}}$ from the experimental data. Here $\rho_{0}$ is the fitting parameter and corresponds to average resistivity of the diffusion zone, which is unknown and is usually no uniform. The value of the diffusion length $l_{\mathrm{d}} \approx 0.22-$ $1600 \mu \mathrm{m}$, evaluated in the same manner for the standard firing conditions is essentially higher than the main distance between dopant particles

$$
L=2 r \sqrt[3]{\frac{\pi}{6 C}}=2 r \sqrt[3]{\frac{\pi}{6}\left[1+\frac{\gamma_{\mathrm{d}}}{\gamma_{g}}\left(\frac{1}{C_{m}}-1\right)\right]}
$$

From (6) we have $L \simeq 0.16 \mu \mathrm{m}$ for main diameter $2 r \approx 0.1 \mu \mathrm{m}$ of dopant particles and volume fraction $C \approx 0.16$, so diffusion zones are heavily overlapped and the whole volume of the specimen is uniformly filled by infinite conductive cluster, as shown schematically in Figure 4. There $\gamma_{\mathrm{d}}$ and $\gamma_{\mathrm{g}}$ are the specific weight of dopant and glass accordingly, $C_{\mathrm{m}}$ is the mass fraction of the dopant.
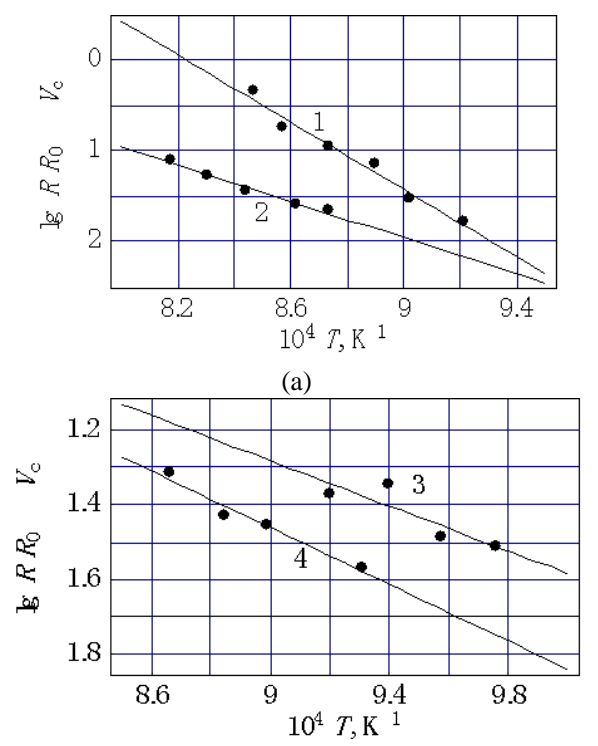

(b)

Figure 3. $R\left(T_{\mathrm{f}}\right)$ for DSG mentioned above (see Introduction). Solid lines are fitting of (5) to experimental data by the least squires method.
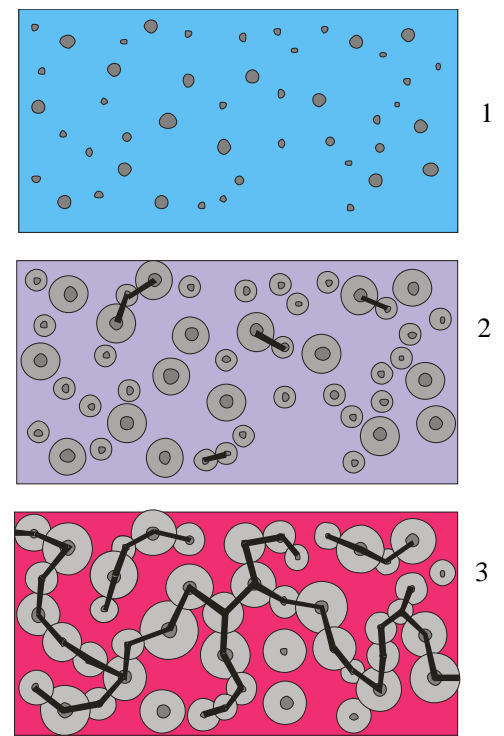

Figure 4. Generation of percolation levels due to diffusion (schematically). The bold curve is the infinite cluster. Gray circles represent diffusion zones. $T_{\mathrm{f}}$ in $\mathbf{3}$ is higher than in $\mathbf{1}$. $\tau=$ const.

Expression (5) shows good agreement with the experimental data for DSG of other compositions [16] as well (Figure 5).

One can also derive the effect of firing duration $\tau$ on the resistivity $\rho$ of DSG from (2) - (4):

$$
\tau=\frac{1}{D\left(T_{\mathrm{f}}\right)}\left[\sqrt[3]{\frac{3}{4 \pi}\left[V_{0}\left(\rho_{0} / \rho\right)^{1 / t}+V_{c}\right]}-r\right]^{2}
$$

This expression is compared with the experiment in Figure 6.

We have simulated the effect of doping level and size of dopant particles on the $\rho$ of DSG. The simulation is based on Fick's equation [17] and expression (5). It is assumed that 1) diffusion from neighbor particles of the dopant into the glass interlayer is uniform; 2) value of $\rho$ is predetermined by minimum of concentration $N$ of dopant atoms at the middle point being at distance $L / 2$ from each of them, i.e.,

$$
\rho \propto N^{-1}(x=L / 2) .
$$

The result of the simulation of $\rho(\tau)$ for two sizes of dopant particles is shown in Figure 7. It is clear on one hand that the size of dopant particles essentially affects on the conductivity of DSG. On the other hand $R(\tau)$ at small $\tau$ is stronger in our simulation than in the usual model of diffusion $R(\tau) \propto \exp (-c \sqrt{\tau})$ (the last is shown in Figure 7 as dotted line).

Resistivity of DSG is strongly affected by the dispersivity of the dopant powder [16] - reduction of the di-ameter of the particles from $4.5 \mu \mathrm{m}$ up to $1.6 \mu \mathrm{m}$ decreases 


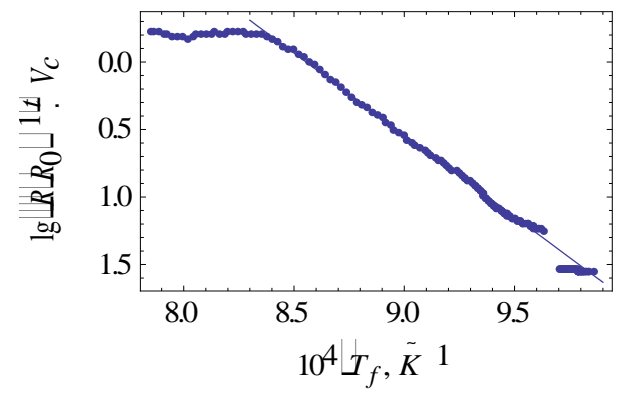

Figure 5. $R\left(T_{\mathrm{f}}\right)$, recalculated data from [16] in accordance with (5). The solid line is fitting of (5) with $R_{0}=10 \mathrm{Ohm}, t=$ $1.7, V_{c}=0.16$.

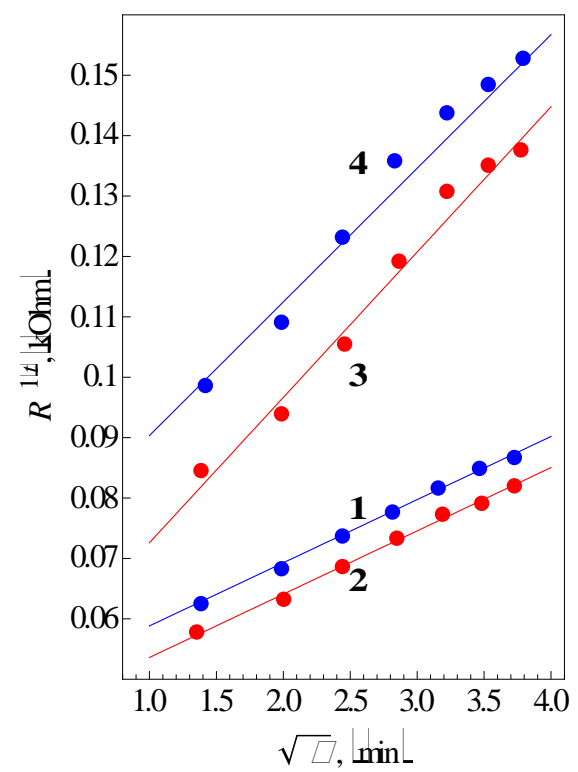

Figure 6. $R(\tau)$ for DSG at $T_{\mathrm{f}}, \mathrm{K}: 1073(1,2)$ and $1123(3,4)$. Temperature increasing rate is higher for data 1 and 2 , than for data 3 and 4 . Points are recalculated from [16] in accordance with (7). DSG composition isn't varied.

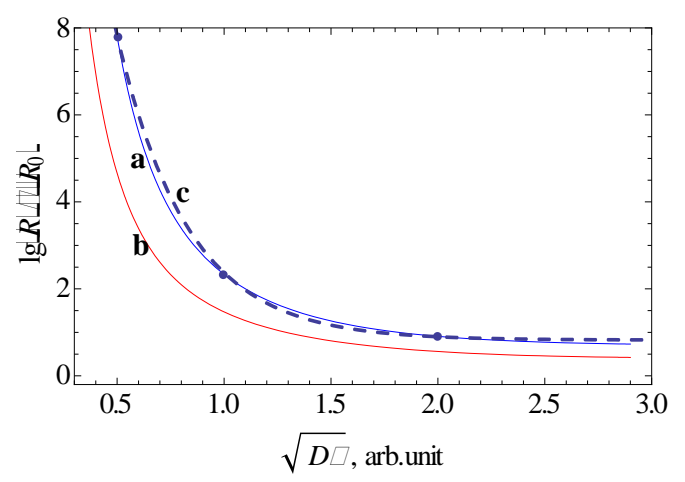

Figure 7. The result of simulation $R(\tau)$ of DSG for $\boldsymbol{r}, \boldsymbol{\mu m}$ : 0.5 (a); 1 (b). $L \approx 5 \boldsymbol{\mu m}$. The function $a+b \exp (-c \sqrt{\tau})$ (dotted line) have been fitted to curve a in the 3 points.

the resistivity by bout one order in magnitude.

We have simulated this effect as the result of diffusion

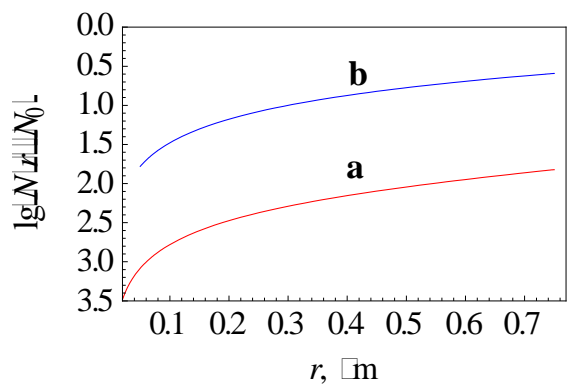

Figure 8. Effect of the main size of dopant particles on concentration of the dopant atoms at the point $x=L / 2=2.5 \boldsymbol{\mu m}$ between the neighbor dopant particles (simulation, see Figures 2 and 7). Content of dopant in DSG is 2 times less for curve a than for curve $b$. The diffusion duration accords to the diffusion length $\sqrt{D \tau}=1,25 \mu \mathrm{m}$.

process (Figure 8) in accordance with the expression (5) for two values of $C<C_{c}$. There we assume that $\rho \propto N^{-1}(x=L / 2)$, the same assumption is made in fig. 7. One can see that the dependence $\rho(r)$ or

$N(r, x=L / 2)$ becomes stronger for smaller $r$, when the main distance between particles of the dopant have been decreased for constant $C$, and conductivity of the DSG increases.

It must be noted here that the information on the solubility of $\mathrm{RuO}_{2}$ in lead-silicate glass is contradictory: Palanisamy et al. [18] estimate it less than $10^{-4}$ atomic \%, while Flachbart et al. [8] present the value about 7 atomic \%. We are inclined to admit the last value because of the results of X-ray diffraction experiments, in which the intensity of main reflexes of $\mathrm{RuO}_{2}$ relicts in DSG reduces nearly $10 \%$ due to firing and they become wider [19]. One should also take into account the fact that solubility of pure metals in silicate glass is very small (really less than $10^{-3}$ atomic\%, [20]), while solubility of their oxides is essentially higher (up to $10-70 \mathrm{~mol}$. \% for $\mathrm{PbO}$ ).

It is clear in the same approach that the electrical properties of DSG, specifically $\rho(T)$, beyond the formation of infinite cluster from diffusion zones, mainly depend on the properties of the doped glass, namely, on the glass structure, on the distribution of the energy levels of the impurity in the energy gap of the glass, and on other microscopic characteristics.

The resistivity of the glass in the diffusion zone is 5 - 6 orders of magnitudes higher than that of the dopant relicts, so the latter one does not affect the macroscopic parameters of the DSG unless they form an infinite cluster (i.e. unless $C<C_{c}$ ). Indeed, the conduction is metallic at $C>C_{\mathrm{c}}$.

\section{Conclusions}

The experiments and simulations show that the diffusion zones are formed around the dopant particles in DSG due 
to the diffusion of dopant atoms into the glass during the firing. The glass becomes conductive in these zones and the overlapping of them generates the infinite conductive cluster (s) (percolation levels). This model allows explaining the effect of technological parameters such as temperature and duration of firing, glass and dopant composition, dispersivity of the powders, on the electrical properties of the DSG as well as the absence of percolation threshold or its shift to smaller values of the dopant content

\section{Acknowledgements}

Fond for Support of Fundamental Researches of the Uzbek Academy of Sciences is acknowledged for the financial support (grants 55-08 and 27-10).

\section{REFERENCES}

[1] H. C. Angus and P. E. Gainsbury, "Glaze Resistors with Ruthenium Dioxide,” Electronic Components, Vol. 9, No. 1, 1968, p. 84.

[2] M. Prudenziati, “Thick Film Sensors,” Elsevier Science, Amsterdam, 1994.

[3] Thick Film Heating Technology, www.watlow.com.

[4] G. E. Pike and C. H. Seager, "Electrical Properties and Conduction Mechanisms of Ru-Based Thick-Film (Cermet) Resistors," Journal of Applied Physics, Vol. 48, No. 12, 1977, p. 5152. doi:10.1063/1.323595

[5] D. P. H. Smith and J. C. Anderson, "Electron Conduction in Thick Film Resistors,” Thin Solid Films, Vol. 71, No. 1, 1980, pp. 79-89. doi:10.1016/0040-6090(80)90186-8

[6] A. Abe and Y. Taketa, "Electrical Conduction in Thick Film Resistors," Journal of Physics D: Applied Physics, Vol. 24, No. 7, 1991, p. 1163.

[7] W. Schoepe, "Conduction Mechanism in Granular $\mathrm{RuO}_{2-}$ Based Thick-Film Resistors,” Physica B: Physics of Condensed Matter, Vol. 165, 1990, pp. 299-300.

[8] K. Flachbart, V. Pavlrk, N. Tomašovičová, C. J. Adkins, M. Somora, J. Leib and G. Eska, "Conduction Mechanism in $\mathrm{RuO}_{2}$-Based Thick Films," Physica Status Solidi (B), 205, No. 1, January 1998, pp. 399-404. doi:10.1002/(SICI)1521-3951(199801)205:1<399::AID-P $\underline{\text { SSB399>3.0.CO;2-X }}$
[9] G. Abdurakhmanov and N. G. Abdurakhmanova, "High Temperature Anomalies in Resistivity and Thermoelectric Power of Thick Film Resistors,” Physica Status Solidi (A), Vol. 202, No. 9, 2005, pp. 1799-1803. doi:10.1002/pssa.200420036

[10] A. A. Appen, "Khimiya stekla (Chemistry of Glass, in Russian) Leningrad,” Khimiya Publishing House, Leningrad Branch, 1974.

[11] V. L. Bonch-Bruevich, et al., "Electron Theory of Disordered Semi Conductors (in Russian),” Nauka Publishers, Moscow, 1981.

[12] B. I. Shklovskii and A. L. Efros, "Electronic Properties of Doped Semiconductors,” Springer, Heidelberg, 1984.

[13] J. Ziman, "Models of Disorder," Cambridge University Press, Cambridge, 1979.

[14] C. Grimaldi, T. Maeder, P. Ryser and S. Strässler, “A Model of Transport Non-Universality in Thick-Film Resistors,” Applied Physics Letters, Vol. 83, 2003, p. 189.

[15] G. Abdurakhmanov and G. S. Vakhidova, "Diffusion and Electrical Conduction in Thick Film Resistors,” Technical Physics, The Russian Journal of Applied Physics, Vol. 65, No. 7, 1995, p. 178.

[16] J. Lee and R. W. West, "Firing Studies with Model Thick Film Resistor Systems,” IEEE Transactions on Components, Hybrids and Manufacturing Technology, Vol. 6, No. 4, 1983, p. 430.

[17] J. P. Stark, “Solid State Diffusion,” Wiley, New York, 1976.

[18] P. Palanisamy, D. H. R. Sarma and R. W. Vest, "Solubility of Ruthenium Dioxide in Lead Borosilicate Glasses," Journal of the American Ceramic Society, Vol. 72, No. 9, September 1989, pp. 1755-1756. doi:10.1111/j.1151-2916.1989.tb06321.x

[19] G. Abdurakhmanov, G. Vakhidova and L. Tursunov, "Interaction of $\mathrm{RuO}_{2}$ and Lead-Silicate Glass in ThickFilm Resistors," World Journal of Condensed Matter Physics, Vol. 1, No. 1, February 2011, pp. 1-5.

[20] H. D. Schreiber, F. A. Settle, P. L. Jamison, J. P. Eckenrode and G. W. Headley, "Ruthenium in Glass-Forming Borosilicate Melts," Journal of the Less Common Metals, Vol. 115, No. 1, January 1986, pp. 145-154. doi:10.1016/0022-5088(86)90379-6 